\title{
OPERATING EXPERIENCE WITH THE NEW TRIUMF RF CONTROL SYSTEM
}

\author{
K. Fong, M. Laverty, S. Fang, TRIUMF, 4004 Wesbrook Mall, Vancouver, B.C., Canada V6T 2A3
}

\section{Abstract}

The $23 \mathrm{MHz}$ rf control of the TRIUMF cyclotron has been replaced by a new VXI control system based on digital signal processing. It provides amplitude and phase regulation of the cyclotron dee voltage, as well as other functions such as power-up sequencing, spark and high VSWR protection. Modularity of the hardware is achieved by the VXI architecture, and in the software by Object Oriented Programming. It is expected that this will result in a considerably longer MTBF, and shorter fault diagnosis and repair times, than the equipment it replaces. The new system has now been in operation for over two months. The results of commissioning, testing, and early operating experience are presented.

\section{INTRODUCTION}

The system described here represents the first major upgrade of the original TRIUMF cyclotron main rf control system [1], and is an outgrowth of earlier systems developed at TRIUMF. Its most recent predecessor is a VME-based system used for control of a $92 \mathrm{MHz}$ booster cavity [2]. Moving to a VXI platform allows much more functionality to be incorporated in each module. Also, the level of shielding inherent to VXI permits the incorporation of low-level analog as well as rf elements in the rack modules. This results in a high level of integration and fringe benefits such as much reduced fault diagnosis and repair times.

Preliminary test results using a resonator test facility were reported at EPAC'94 [3]. This system has now been installed and commissioned in the cyclotron. This paper offers a brief review of the noteworthy features of the new control system, together with data and observations collected in the course of commissioning and early operation.

\section{SYSTEM OVERVIEW}

A photo of the VXI control rack which contains virtually all of the active electronics is shown in Figure 1. Of the 13 available VXI slots, the first two (starting from the left) are occupied by the system controller. This is an embedded PC compatible which provides the VXI-bus interface and timing, the local operator interface, as well as most of the intelligence of the system. The second module, like the first, is a commercially available unit. It provides an interface for the safety interlock signals to the VXI bus. The remaining modules were designed at TRIUMF to meet the requirements of this application. The first of these is the diagnostics module. This module provides analog data capture and data logging from various test points throughout the system via the VXI analog bus. The next module is the amplitude/phase controller. It provides high speed A/D and D/A conversion, as well as a pair of digital signal processors which implement the respective amplitude and phase control algorithms. The DSP's are programmed for Proportional-Integral-Differential feedback and run autonomously. Communication between the DSPs and the VXI interface is needed only when updating the PID parameters, and opening and closing the feedback loop.

Next to the amplitude/phase controller and coupled to it via the backplane is the rf module. This is the module where most of the low-level rf processing takes place. It includes the amplitude/phase modulator, the amplitude and phase detectors, and an rf switch to select between driven and selfresonant modes of operation. From the rf module, the rf signal goes to the preamp module. Here the signal is amplified to a level of about $5 \mathrm{~W}$ before being output to the driver amplifiers. This module also performs VSWR detection, generating an interrupt to the DSP module when the reflected power exceeds a programmed limit. The direct digital frequency synthesizer module resides in the next slot, and provides a program controlled reference frequency for the driven mode of operation. Direct digital synthesis was chosen due to its ability to change frequency without generating amplitude and phase glitches. The frequency can also be set via remote or local soft pots.

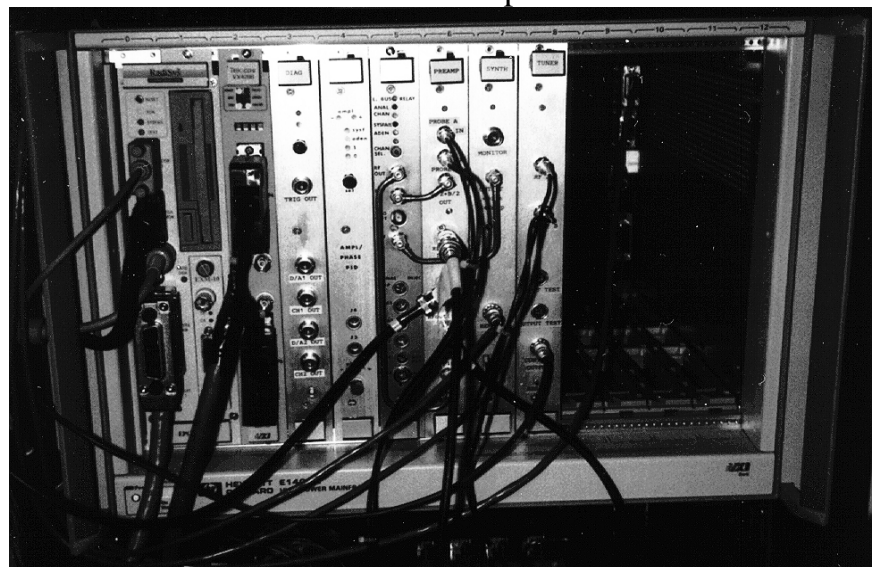

Figure 1 - VXI System Rack

The next module, the tuner controller, also uses a DSP. In this case convenience, rather than bandwidth, is the main 
consideration. The tuning system uses modulation of the cooling water pressure to effect a change in resonator frequency, and has a bandwidth of the order of $1 \mathrm{~Hz}$. . The last of the modules is the AIDC [4]. For the purposes of this system, it provides SDLC communications with the control room computers.

The system software was designed using the objectoriented constructs of the $\mathrm{C}++$ language. A generic VXI interface is used as a parent object for all the register-based VXI modules. Higher level objects such as DSP interfacing inherit the functionality of this VXI object. Together with additional communication and control software for the DSP, it forms the parent for the three different DSP module interfaces. The state of every hardware module is updated every $0.05 \mathrm{sec}$ and, for diagnostic purposes, this can be displayed in a dialog window. All the relevant information and controls necessary for normal running are duplicated and grouped into one single dialog window. The same information is sent to the central control system via the AIDC module, and displayed in an $\mathrm{X}$-window on the operator console. Commands from the central control system are interpreted and acted upon. State changes and their causes are sent to a printer for logging.

\section{SYSTEM INTEGRATION}

Installation of the new control system was arranged to take place about one week prior to a planned cyclotron shutdown. This allowed for comparative testing with the old rf system, without the complications of shutdown related problems. Initial testing was accomplished in a series of stages, beginning with low power open-loop operation, and continuing on to full power close-loop operation. A control system analyzer was employed to measure parameters such as loop gain and noise spectra under open and closed loop conditions. A sample of the measured open loop frequency response is shown in Figure 2. The resulting curve shows almost single pole behavior, where the loop gain falls off at $20 \mathrm{~dB} /$ decade, until the unity gain point is reached. The low frequency pole is due to the integral component of the control algorithm. A second pole is located at about $3 \mathrm{kHz}$, which is due to the bandwidth of the cyclotron resonator. This pole is canceled by a zero at about $8 \mathrm{kHz}$, which arises from the proportional component of the control algorithm. The system group delays begin to dominate above $20 \mathrm{kHz}$. Loop gain at $10 \mathrm{~Hz}$ exceeds $60 \mathrm{~dB}$. Phase margin is $130^{\circ}$ at $9 \mathrm{kHz}$, and gain margin is $20 \mathrm{~dB}$ at $80 \mathrm{kHz}$. If necessary, the integral gain can be increased by factors of more than 10 to improve regulation below $3 \mathrm{kHz}$, and the proportional gain can be increased by a factor of 3 to improve regulation above $3 \mathrm{kHz}$, and still have sufficient gain and phase margin for stability.

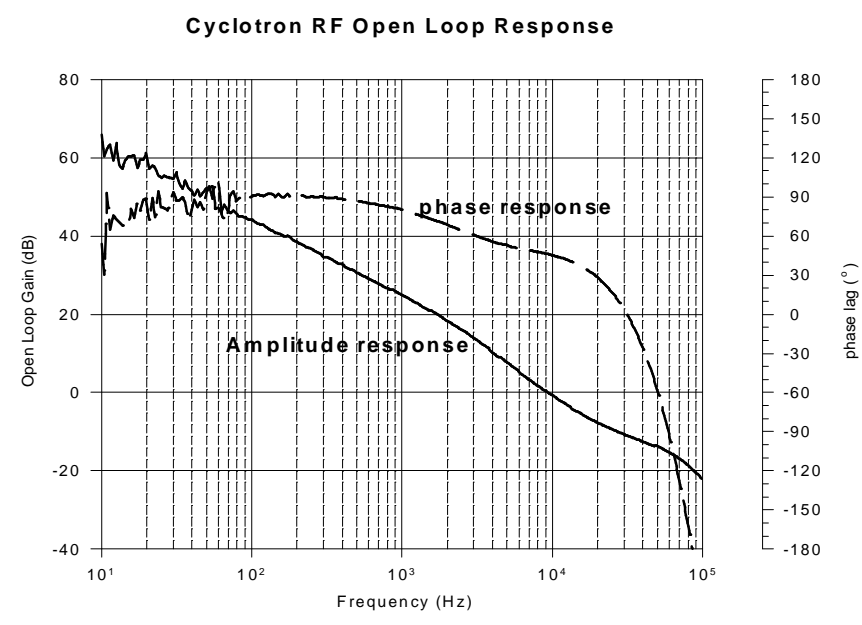

Figure 2 - Amplitude Loop Frequency Response

Similar measurements of the tuner control system produced the curve of Figure 3. The mechanics of this system result in a number of poles located at about $1 \mathrm{~Hz}$. This puts a severe upper limit on the bandwidth which can be achieved, but does not cause any actual problems in practice, as this system's main purpose is to deal with thermal drift of the resonator frequency.

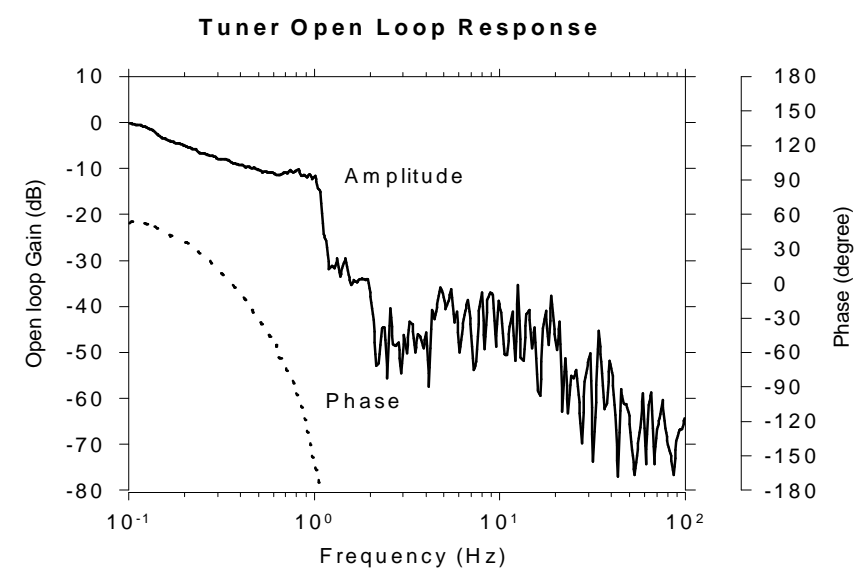

Figure 3 - Tuner Loop Response

Final test and integration had to await the end of the shutdown. This included testing of the control room operator interface software, as well as further testing and optimizing of the controls. One item in particular which had to wait for rf startup was optimization of the spark detector. The spark detector monitors the rf level, its fall time, as well as the reflected power in the main transmission line. This detector operates on the log of the resonator voltage. If the resonator voltage drops below $10 \mathrm{kV}$ for more than $10 \mu \mathrm{s}$, the drive is shut off. This is classified as a 'level trip'. Figure 4 shows an example of a level trip after the dee voltage has been decimated by a medium spark. The log amplitude signal is 
also differentiated, and the resulting signal passed to three comparators. These are adjusted to detect three different $\mathrm{rf}$ decay rates, corresponding to small, medium, and large sparks in the resonator. Figure 5 shows the relative magnitude of these signals and their associated fall times. If the fall time of the dee voltage is such that it drops to less than $10 \%$ of its nominal value in less than $4 \mu \mathrm{s}$, it is classified as a 'large spark trip' and the drive is shut off. The power amplifier is also protected against high reflected power. If the output of the reflected power detector in the preamp module exceeds a safe threshold value, the preamp module interrupts the DSP module via a local bus line. The amplitude DSP responds by reducing the output drive by a predetermined step and opening the feedback loop, if it is closed. As long as the reflected power exceeds the threshold, the output drive continues to decrease. If the problem corrects itself, the controller can rapidly ramp the drive up to full power again.

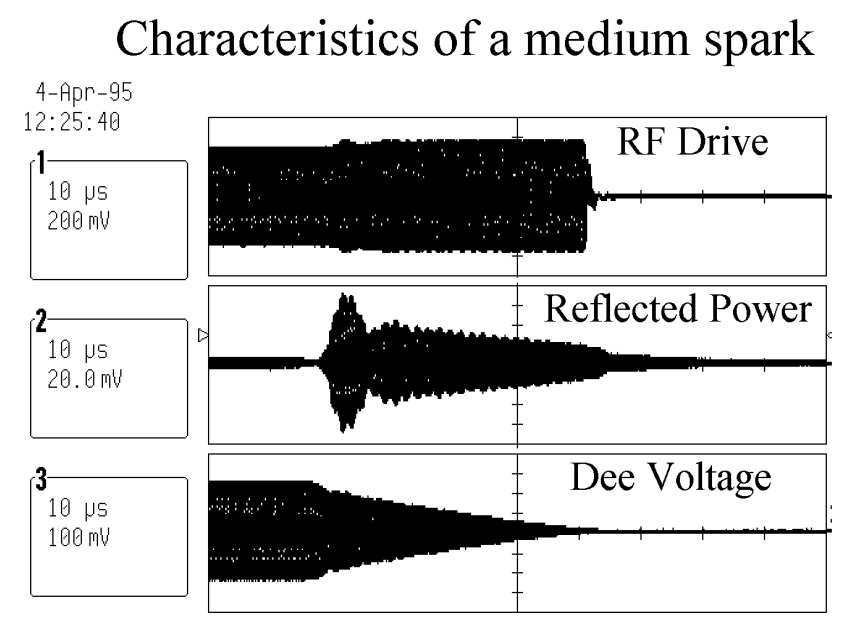

Figure 4 - Medium Spark

\section{CONCLUSION}

Owing to careful preparatory work, the installation of the new system went relatively smoothly, considering the magnitude of the changes. As a precaution, the original system was left intact and ready to be reconnected if required. This option was not needed, however, and no significant downtime has been attributed to the new controls or the minor changes which have been required to date. As an example, one change which proved necessary was to increase the gain parameters to accomodate the effects of beam loading at high beam currents. This was readily accomplished via the local operator interface without interrupting the operation of the rf or the beam. Further work is being done to reduce the downtime of the system by fine tuning of the spark detection parameters, better diagnostic data logging, and improved data exchange with remote computers.

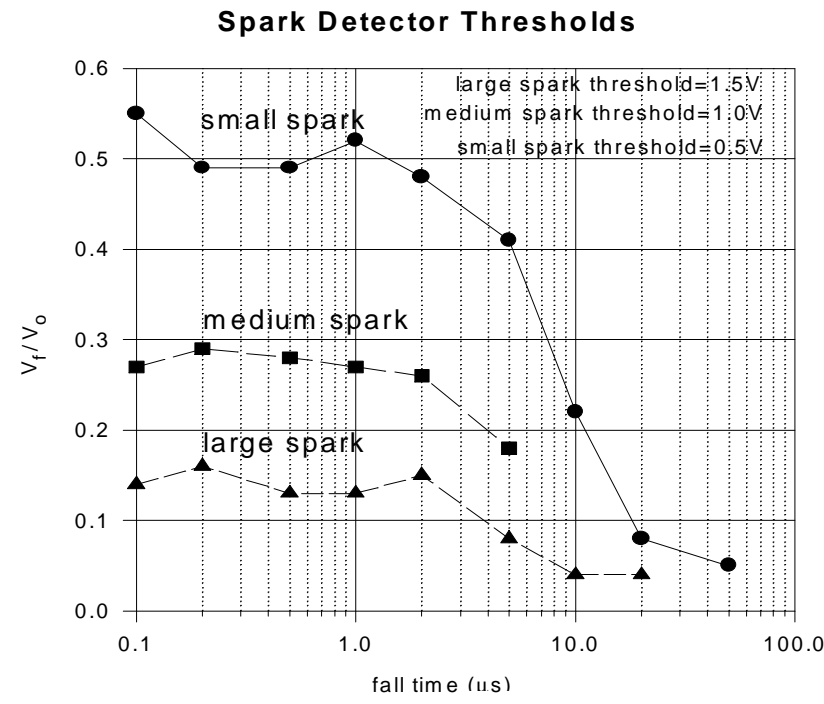

Figure 5 Spark Detector Thresholds

\section{REFERENCES}

[1] R.H.M. Gummer, "RF Accelerating Voltage Control and Stabilization in the TRIUMF Cyclotron", IEEE Transactions on Nuclear Science, NS-22, No. 3, 1257, June 1975.

[2] K. Fong, M. Laverty, S. Fang, "RF Control System Upgrade at Triumf", European Particle Accelerator Conference Proceedings, London, June 1994, pp. 18421844.

[3] K. Fong, M. Laverty, "RF Control System for TRIUMF Booster Cavity", European Particle Accelerator Conference Proceedings, Berlin, Mar 1992, pp. 11761178.

[4] D. Bishop, G. Waters, D. Dale, T. Ewart, D. Harrison, J. Lam, R. Keitel, "Development of an autonomous 32-bit intelligent device controller", Proceedings of the Third International Conference on Accelerator and Large Experimental Physics Control Systems, Berlin, Oct 1993, pp. 236-238. 